\title{
Diacronie
}

Studi di Storia Contemporanea

$\mathrm{N}^{\circ} 29,1$ | 2017

"Crash test"

\section{Riflessioni sulla narrazione storica nelle voci di Wikipedia}

Tommaso Baldo

\section{(2) OpenEdition}

Journals

\section{Edizione digitale}

URL: http://journals.openedition.org/diacronie/5347

DOI: $10.4000 /$ diacronie.5347

ISSN: 2038-0925

\section{Editore}

Association culturelle Diacronie

Notizia bibliografica digitale

Tommaso Baldo, «Riflessioni sulla narrazione storica nelle voci di Wikipedia », Diacronie [Online], №

29, 1 | 2017, documento 6, Messo online il 29 mars 2017, consultato il 19 avril 2019. URL : http://

journals.openedition.org/diacronie/5347 ; DOI : 10.4000/diacronie.5347 


\title{
Diacronie
}

Studi di Storia Contemporanea

29, $1 / 2017$

"Crash test". Continuità, discontinuità, legami e rotture nelle dinamiche della storia contemporanea

\section{Riflessioni sulla narrazione storica nelle voci di Wikipedia}

\author{
Tommaso BALDO
}

in collaborazione con il gruppo di lavoro Nicoletta Bourbaki

Per citare questo articolo:

BALDO, Tommaso, «Riflessioni sulla narrazione storica nelle voci di Wikipedia», Diacronie. Studi di Storia Contemporanea : "Crash test". Continuità, discontinuità, legami e rotture nelle dinamiche della storia contemporanea, 29, $1 / 2017,29 / 3 / 2017$,

URL: < http://www.studistorici.com/2017/03/29/baldo_numero_29/ >

Diacronie Studi di Storia Contemporanea $\rightarrow$ http://www.diacronie.it Rivista storica online. Uscita trimestrale.

redazione.diacronie@hotmail.it

Comitato di direzione: Naor Ben-Yehoyada - João Fábio Bertonha - Christopher Denis-Delacour - Maximiliano Fuentes Codera Anders Granås Kjøstvedt - John Paul Newman - Deborah Paci - Niccolò Pianciola - Spyridon Ploumidis - Wilko Graf Von Hardenberg

Comitato di redazione: Jacopo Bassi - Luca Bufarale - Gianluca Canè - Fausto Pietrancosta - Alessandro Salvador - Matteo Tomasoni Diritti: gli articoli di Diacronie. Studi di Storia Contemporanea sono pubblicati sotto licenza Creative Commons 3.0. Possono essere

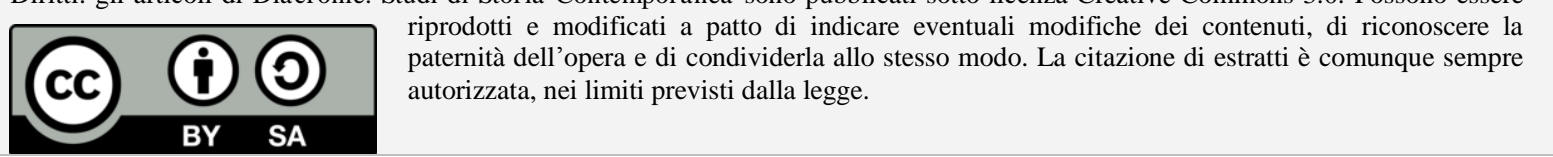




\title{
6/ Riflessioni sulla narrazione storica nelle voci di Wikipedia
}

\author{
Tommaso BALDO ${ }^{1}$
}

Quale rapporto si sta costruendo fra le scienze storiche e Wikipedia? Come bisogna porsi di fronte alle voci di Wikipedia che trattano di storia? Quale potrà essere il ruolo della futura "enciclopedia libera" per lo studio e l'insegnamento della storia? A partire dall'analisi di alcune voci di Wikipedia operata dal gruppo di lavoro "Nicoletta Bourbaki", sei autori animano una tavola rotonda sul rapporto tra l'enciclopedia libera e il mondo della storia.

Un'analisi delle voci storiche di Wikipedia non può prescindere dal problema del «chi» scrive le sue voci. In un suo articolo lo storico Giovanni De Luna esprimeva una certa preoccupazione proprio in merito al fatto che gli autori delle narrazioni storiche più consultate sul web non siano figure riconosciute e riconoscibili:

L'analisi (elaborata da Barbara Montesi) dei due siti più frequentati dagli studenti (Wikipedia $\mathrm{e}$ Cronologia) delinea una situazione preoccupante. In Wikipedia si dissolve uno dei requisiti essenziali della ricerca storica: la verificabilità del dato attraverso la certezza dell'identità del suo autore. Nel web, infatti, tutti possono scrivere di storia, accreditahersi come storici: genealogisti e antiquari, cultori delle memorie familiari e storici locali, professionisti e dilettanti; si dissolvono le tradizionali gerarchie accademiche, nasce una nuova comunità enormemente allargata fuori dall'università, dalle riviste, dalle fondazioni e dalle altre istituzioni che erano stati da sempre $\mathrm{i}$ «luoghi» esclusivi della ricerca ${ }^{2}$.

De Luna coglie un dato centrale: a scrivere sull'«Enciclopedia libera» non è un gruppo di lavoro ben definito e neppure vi è qualche istituzione o «gruppo di esperti» che si assuma la responsabilità

\footnotetext{
${ }^{1}$ In collaborazione con il gruppo di lavoro Nicoletta Bourbaki.

2 DE LUNA, Giovanni, «Tutti sono storici, ma chi è Tacito?», in La Stampa, 8 maggio 2012, URL: <http://www.lastampa.it/2012/05/08/cultura/libri/tutti-sono-storici-ma-chi-e-tacitoxwEE0ACi9CYAwy2FjFXHrN/pagina.html > [consultato il 16 settembre 2016].
} 
di quanto vi si può leggere. Vi è piuttosto uno spazio in continua evoluzione in cui singoli (e in realtà anche gruppi) interagiscono in modo più o meno conflittuale cercando di rendere egemone la propria visione del passato.

Vi è però anche un altro aspetto da sottolineare. La dissoluzione «delle tradizionali gerarchie accademiche» crea per l'appunto «una nuova comunità». Ma come tutte le comunità anche quella dei «wikipediani» ha le proprie regole, le proprie gerarchie, i propri conflitti e soprattutto i propri confini.

Non siamo quindi in presenza di un generico «tutti» contrapposto all" «accademia», ma di una determinata comunità e, se parliamo della sezione in italiano di Wikipedia, forse persino di una comunità più ristretta di quella dei tradizionali divulgatori del sapere storico (operatori museali, autori di saggi ed articoli, docenti universitari e della scuola dell'obbligo, eccetera). Sono infatti una piccola minoranza, al massimo alcune migliaia di persone, gli utenti (registrati o meno) di it.Wiki che vengono considerati «attivi», vale a dire coloro che hanno effettuato cinque modifiche in un mese ad una o più voci3.

Il ristretto numero degli utenti «attivi» condiziona molte voci, ad esempio alcuni argomenti divengono praticamente monopolio di una o poche persone, le uniche ad occuparsene. Oppure i rapporti interpersonali (simpatie, antipatie, affinità, ecc.) finiscono per acquisire un peso determinante nelle dinamiche interne.

Occorre aggiungere che le regole alla base dell'«enciclopedia libera» («i cinque pilastri») sono abbastanza vaghe da rendere possibili una vasta gamma di interpretazioni. Si limitano infatti a raccomandare: il valore enciclopedico di ogni voce creata; la ricerca del «punto di vista neutrale» (Neutral point of view o Npov) attraverso la presentazione di tutti i punti di vista su un argomento; la libera partecipazione nella scrittura delle voci e nella circolazione dei contenuti presenti su Wikipedia; il rispetto degli altri utenti attraverso la ricerca della collaborazione; l'assenza di regole fisse al di fuori dei cinque pilastri ${ }^{4}$. Ciascuna sezione nelle diverse lingue può poi elaborare le proprie linee guida variabili.

A vegliare sul rispetto delle regole sono gli amministratori, $i$ volontari scelti in seguito a votazione tra gli utenti registrati con maggiore esperienza e che hanno editato il maggior numero di contributi (almeno 500) alle voci di Wikipedia. Possono essere riconfermati per modalità tacita (solo se vi sono utenti che si dichiarano contrari si procede alle votazioni) dopo un anno dalla prima attribuzione delle funzioni o dall'ultima riconferma ${ }^{5}$. Ovviamente i rapporti interpersonali e gli

\footnotetext{
${ }^{3}$ TALIA, Salvatore, «Fascinazione Wikipedia, il mito della 'cricca' e il conflitto reale», in Giap, URL:

$<$ http://www.wumingfoundation.com/giap/?p=17162 > [consultato il 12 luglio 2016].

${ }^{4}$ «Wikipedia: I cinque pilastri», in Wikipedia, URL:

< https://it.wikipedia.org/wiki/Wikipedia:Cinque_pilastri > [consultato il 12 luglio 2016].

${ }^{5}$ «Wikipedia: Amministratori», in Wikipedia, URL:

< https://it.wikipedia.org/wiki/Wikipedia:Amministratori\#Riconferma_annuale > [consultato il 12 luglio
} 
equilibri all'interno della non vastissima comunità dei wikipediani italiani finiscono per avere un peso anche nella scelta di questi «arbitri».

Veniamo infine ai conflitti all'interno della comunità. Ogni gruppo umano sarà sempre diviso al proprio interno da differenti ideali, interessi, simpatie, antipatie, eccetera. Questa realtà si scontra però con la grande aspirazione di Wikipedia, ovvero la pretesa di considerare il «Punto di vista neutrale» uno dei propri «cinque pilastri», e quindi un «valore non negoziabile». Proprio per questo non si possono scrivere su Wikipedia ricerche originali su un argomento, cioè testi che si basino su una propria ricerca e non sul sunto di studi altrui. Le ricerche originali sono considerate incompatibili con il concetto di «enciclopedia» e non possono essere esposte in modo neutrale.

In teoria bisognerebbe quindi esporre le dispute senza prendervi parte, cercando di presentare in modo imparziale tutti gli aspetti di una questione. Come possiamo leggere sulla stessa Wikipedia: «Il Punto di vista neutrale richiede che una voce illustri correttamente tutti i punti di vista significativi che sono stati descritti da fonti attendibili, e che debba farlo in misura proporzionata allimportanza di ciascuno» ${ }^{6}$.

Ma come si arriva al «Punto di vista neutrale»? L'«enciclopedia libera» non prevede un comitato scientifico di esperti né pratiche di peer review. Pertanto il «Punto di vista neutrale» viene continuamente rinegoziato tra gli utenti stessi, come si può vedere facilmente scorrendo qualunque pagina di «discussione» in cui vi sia qualche dibattito e seguendo l'alternarsi dei contributi in una voce nella «cronologia».

Dunque siamo in presenza di una narrazione storica costruita di fatto da chi riesce a raccogliere maggior consenso nella comunità degli utenti e degli amministratori di Wikipedia. Spesso si tratta di chi ha più tempo, una risorsa fondamentale da spendere per presidiare le voci e per acquisire credito ed esperienza all'interno della comunità dei wikipediani attraverso la propria mole di contributi.

Inoltre nella stesura di una voce preverrà il punto di vista di quell'utente, o di quel gruppo di utenti, che dimostrerà più volontà/capacità di gestire polemiche a volte estenuanti (al punto da allontanare gli utenti solo occasionalmente «attivi») e che più a lungo conserverà la motivazione che lo spinge a «presidiare» per mesi ed anni una o più voci.

A questa particolare declinazione delle storture della «democrazia della rete» si aggiunge una pratica di utilizzo delle fonti che non può che favorire distorsioni e anche falsificazioni palesi. Se «fontato» con una nota a piè di pagina un testo può essere modificato solo con molte difficoltà. Ovvero bisogna intervenire nella pagina di «discussione», dimostrare che la fonte è citata in modo errato o improprio, oppure bisogna addurre una o più fonti contrarie, far si che siano accettate come

2016].

${ }^{6}$ «Wikipedia: Punto di vista neutrale», in Wikipedia, URL:

< https://it.wikipedia.org/wiki/Wikipedia:Punto_di_vista_neutrale > [consultato il 12 luglio 2016]. 
più autorevoli e solo quando si sarà ottenuto il consenso degli amministratori e degli altri utenti che partecipano alla discussione si potrà modificare il testo. Ovviamente questa pratica favorisce l'utilizzo delle fonti come «cavalli di frisia nelle guerre di posizione wikipediane»"

Le analisi di diverse voci di it.Wiki, soprattutto tra quelle dedicate a temi «sensibili» quali la storia della Resistenza e quella delle zone di confine, svolte dal gruppo di lavoro Nicoletta Bourbaki e pubblicate sul blog Giap hanno portato alla luce diversi esempi di uso distorto e parziale delle fonti, nonché di vere e proprie falsificazioni. Ad esempio si è notato l'uso dei testi di Arrigo Petacco e Indro Montanelli come pezze d'appoggio per diffondere falsi storici sulle foibe, oppure si è segnalato uno strafalcione di Bruno Vespa usato per falsificare la biografia di Franco Basaglia (cui veniva attribuita addirittura l'arruolamento nelle fila delle truppe del'RSI!) $)^{8}$. Altre volte ancora si è scelto di dare a ricostruzioni nettamente propagandistiche lo stesso peso che si dà a testi storiografici. È il caso ad esempio delle citazioni del pubblicista neofascista Giorgio Pisanò, cui nella voce sull'attacco partigiano di Via Rasella si dà lo stesso spazio che alla storiografia sull'argomento. Senza dimenticare la pura e semplice invenzione di un libro inesistente Assassini nella storia, scritto da un altrettanto inesistente autore, tale Samuel Frederick J. che si voleva pubblicato da una tipografia specializzata in realtà in materiale pubblicitario e non ancora aperta nel 1994, anno della pretesa edizione dell'opera fantasma. Questo testo inesistente serviva a presentare l'organizzazione antifascista degli sloveni della Venezia Giulia, il Tigr, come una semplice accozzaglia di assassini. Per poter dimostrare agli utenti ed amministratori di it.Wiki l'inesistenza dell'autore e del libro sono stati necessari mesi di ricerche e persino la visura camerale per chiarire lo status della presunta casa editrice ${ }^{10}$.

Nella voce «storia del Trentino» ho personalmente riscontrato invece l'utilizzo e la citazione di un pamphlet interventista del 1914 come descrizione della vita politica ed economica nel Trentino prima della Grande guerra. Insomma l'utilizzo di una fonte primaria come se fosse una fonte secondaria, la propaganda di un determinato periodo storico presentata come ricostruzione dello stesso $^{11}$.

I meccanismi di selezione ed utilizzo delle fonti su it.Wiki assomigliano ad una parodia di quelli accademici, una parodia che spesso si dimostra funzionale alla trasmissione di un preciso frame

\footnotetext{
${ }^{7}$ FILIPAZ Lorenzo, «Wi-chi? Battaglie per il sapere in rete», in Zapruder, storie in movimento, 39, 1/2016, pp. 142-149, p. 146.

${ }^{8}$ BOURBAKI, Nicoletta, «Wikipedia e la storia deturpata, il caso Presbite», in Giap URL:

< http://www.wumingfoundation.com/giap/?p=19327\#gilas > [consultato il 12 luglio 2016]

${ }^{9}$ TALIA, Salvatore, «Un paese di 'mandolinisti'. Wikipedia, i falsi storici su via Rasella e il giustificazionismo sulle Fosse Ardeatine», in Giap, URL: < http://www.wumingfoundation.com/giap/?p=21098 > [consultato il 12 luglio 2016]

${ }^{10}$ TALIA, Salvatore, «Fascinazione Wikipedia, il mito della 'cricca' e il conflitto reale», in Giap, URL:

$<$ http://www.wumingfoundation.com/giap/?p=17162 > [consultato il 12 luglio 2016].

${ }^{11}$ BALDO, Tommaso, «I ' 45 cavalieri di Wikipedia', da chi e da che cosa è libera l'enciclopedia libera?», in Giap, URL: < http://www.wumingfoundation.com/giap/?p=22562 > [consultato il 12 luglio 2016].
} 
etnico-nazionalista che narra ogni conflitto delle aree di confine secondo l'esclusiva chiave di lettura dell'odio «atavico» tra popoli diversi, per loro natura impossibilitati a convivere ${ }^{12}$. Naturalmente, la narrazione dei conflitti in questione vede gli italiani nel ruolo di vittime e gli altri popoli in quello di aggressori, persecutori o terroristi, come si può notare sia nelle voci sulla storia del confine orientale che in quelle dedicate alle vicende di Trentino e Alto Adige/Südtirol.

Questo frame etno-nazionalista è favorito dalla natura conservatrice della narrazione del passato messa in campo da Wikipedia nelle sue voci di lingua italiana. Non si tratta di un conservatorismo politico-ideologico, ma storiografico. Il motivo di questo conservatorismo è semplice. Nelle voci dell'«Enciclopedia libera», il dibattito verte su argomenti che fanno riferimento ad una visione del passato consolidata nell'opinione pubblica e alle fonti della vulgata mainstream diffusa dalla stampa quotidiana e dalle trasmissioni della Tv generalista, non al dibattito storiografico. Le voci dell'«Enciclopedia libera» in sostanza si possono considerare spesso frutto di una visione della storia che potremmo definire «pre-annales», ovvero una visione della storia che mette al primo posto gli aspetti politico-militari e diplomatici, anziché quelli legati alla storia economica, sociale, di genere, della mentalità, della cultura e vita materiale. La voce «storia del Trentino» credo sia un esempio lampante di questa distanza tra il frame narrativo alla base di molte voci di it.Wiki e la storiografia più recente in merito agli argomenti trattati.

Alla luce di tutto ciò credo che la migliore descrizione di Wikipedia sia quella data da Lorenzo Filipaz (anch'egli all'interno del gruppo di lavoro Nicoletta Bourbaki) in un suo articolo pubblicato su Zapruder:

Wikipedia non è un'enciclopedia, è una impresa collettiva eternamente incompiuta di scrittura di un'enciclopedia; ed è anche un social network. Per queste ragioni assomiglia più ad una piazza che a un archivio e, come tale, è una lente sui processi culturali e sociali in corso; non tanto uno specchio della società, quanto di quelle minoranze che in buona o cattiva fede si industriano a istruirla gratuitamente ${ }^{13}$.

Mettere in evidenza i difetti ed i limiti di Wikipedia non significa però demonizzarla. Il rifiuto per l'«enciclopedia libera» che porta molti studiosi, educatori e docenti a rifuggire qualunque tipo di rapporto con lei, così come il rifiuto di prendere atto del problematiche sollevate dalle narrazioni storiche sul web, non serve ad altro che a lasciare campo libero ai mistificatori e a favorire l'abbassamento del livello del dibattito.

\footnotetext{
${ }^{12}$ FILIPAZ Lorenzo, op. cit.

${ }^{13}$ FILIPAZ Lorenzo, op. cit., p. 148.
} 
Occorre invece, da un lato un'azione di analisi pubblica di quanto avviene su Wikipedia come quella che abbiamo messo in atto come gruppo di lavoro Nicoletta Bourbaki, dall'altro un forte investimento educativo che abbia la scuola, le biblioteche ed i musei come protagonisti.

Oggi la forza pervasiva del web, la sua capacità di offrire sempre e ovunque una mole potenzialmente infinita di informazioni non filtrate rende del tutto anacronistica ed insufficiente una didattica della storia basata sulla semplice trasmissione di conoscenze. Occorre invece una didattica della storia che si ponga tra i propri obiettivi prioritari la trasmissione di quello che Marc Bloch chiamava «il metodo critico», ovvero una metodologia razionale tramite cui rapportarsi con le fonti alle quali ci rivolgiamo per avere informazioni, valutandone attendibilità e limiti ${ }^{14}$.

Wikipedia può essere il terreno sul quale sperimentare attività didattiche basate sull'individuazione e l'analisi delle criticità contenute nelle sue voci. Per fare questo occorre però capire la portata del cambiamento che il web ha introdotto nella trasmissione delle conoscenze storiche e voler raccogliere le sfide che esso ci pone.

${ }^{14}$ BLOCH, Marc, Apologia della storia o Mestiere di storico, Torino, Einaudi, 2009. 


\section{L'AUTORE}

Tommaso BALDO ha conseguito la laurea Specialistica in Storia d'Europa nel 2009 presso l'Alma Mater Studiorum, Università di Bologna conseguendo la votazione di 110 e lode. Dal maggio 2010 lavora presso la Fondazione Museo storico del Trentino come addetto alla progettazione e realizzazione di attività didattiche. Si è occupato di Wikipedia nel post I «45 cavalieri» di Wikipedia. Da chi e cosa è libera l'enciclopedia libera?, in collaborazione con il gruppo di studio Nicoletta Bourbaki (http://www.wumingfoundation.com/giap/?p=22562) e nell'articolo «L'Arena delle narrazioni. La storia nel tempo di Wikipedia», in Altre Storie, 17, 50, 2/2016, pp. 36-37.

URL: < http://www.studistorici.com/progett/autori/\#Baldo > 